\title{
Potential Synergetic Use of GNSS-R Signals to Improve the Sea-State Correction in the Sea Surface Salinity Estimation: Application to the SMOS Mission
}

\author{
Roberto Sabia, Student Member, IEEE, Marco Caparrini, Adriano Camps, Senior Member, IEEE, and Giulio Ruffini
}

\begin{abstract}
It is accepted that the best way to monitor sea surface salinity (SSS) on a global basis is by means of $\mathrm{L}$-band radiometry. However, the measured sea surface brightness temperature $\left(T_{\mathrm{B}}\right)$ depends not only on the SSS but also on the sea surface temperature (SST) and, more importantly, on the sea state, which is usually parameterized in terms of the 10 -m-height wind speed $\left(U_{10}\right)$ or the significant wave height. It has been recently proposed that the mean-square slope (mss) derived from global navigation satellite system (GNSS) signals reflected by the sea surface could be a potentially appropriate sea-state descriptor and could be used to make the necessary sea state $T_{B}$ corrections to improve the SSS estimates. This paper presents a preliminary error analysis of the use of reflected GNSS signals for the sea roughness correction and was performed to support the European Space Agency's Soil Moisture and Ocean Salinity (SMOS) mission; the orbit and parameters for the SMOS instrument were assumed. The accuracy requirement for the retrieved SSS is 0.1 practical salinity units after monthly averaging over $2^{\circ} \times 2^{\circ}$ boxes. In this paper, potential improvements in salinity estimation are hampered mainly by the coarse sampling and by the requirements of the retrieval algorithm, particularly the need for a semiempirical model that relates $T_{\mathrm{B}}$ and mss.
\end{abstract}

Index Terms-Auxiliary data, global navigation satellite system-reflections (GNSS-R) signals, mean-square slope (mss), microwave radiometry, sea salinity retrieval.

\section{INTRODUCTION}

$\mathbf{T}$ HE SOIL Moisture and Ocean Salinity (SMOS) mission was selected in May 1999 by the European Space Agency (ESA) to provide global and frequent soil moisture and sea surface salinity (SSS) maps. SMOS has a sun-synchronous polar dawn-dusk orbit [1]. Its single payload is the Microwave Imaging Radiometer by Aperture Synthesis (MIRAS), a novel L-band radiometer that makes use of 2-D aperture synthesis interferometry to measure the brightness temperature $\left(T_{\mathrm{B}}\right)$ at two orthogonal polarizations within a wide field of view (FOV)

Manuscript received May 31, 2006; revised March 13, 2007. This work was supported in part by the Spanish Ministry of Science and Education and in part by EU Feder project "Técnicas interferométricas, polarimétricas y biestaticas para sistemas de teledetección por microondas," Ref. TEC2005-06863-C02-01.

R. Sabia and A. Camps are with the Department of Signal Theory and Communications, Universitat Politècnica de Catalunya, 08034 Barcelona, Spain (e-mail: roberto.sabia@tsc.upc.edu).

M. Caparrini and G. Ruffini are with the STARLAB, Barcelona S.L., Edifici de l'Observatori Fabra, Camí de l'Observatori s/n, 08035 Barcelona, Spain.

Digital Object Identifier 10.1109/TGRS.2007.898257 and without any mechanical antenna movement. A particular feature of the MIRAS instrument is its multiangular imaging capability as the satellite moves over the Earth.

At L-band, the $T_{\mathrm{B}}$ over the ocean mainly depends on three variables: the SSS to be measured, the sea surface temperature (SST), and the sea state, which is the largest contributor to the deviations of the brightness temperature with respect to the flat sea model [2]. Often, the sea-state impact on $T_{\mathrm{B}}$ is estimated using the $10-\mathrm{m}$-height wind speed $\left(U_{10}\right)$, the significant wave height (SWH) [3], or both [4], but at L-band, none of these approaches is fully satisfactory.

Furthermore, recent studies have shown that using different sources of auxiliary data (mainly $U_{10}$, as the most readily available parameter to account for sea state) leads to different biases and standard deviations in the retrieved salinity fields [5], in addition to the fact that time and space collocation are sometimes critical.

The potential use of global navigation satellite systemsreflections (GNSS-Rs) opportunity signals for altimetry [6] and sea-state determination in terms of the mean-square slope (mss) has been already tested from ground-based [7]-[10], airborne [11]-[18] and spaceborne [19], [20] experiments. However, the underlying science needs further refinements to extract meaningful physical quantities that can be successfully used in the remote sensing and oceanographic communities.

The potential synergy between GNSS-R mss measurements and L-band radiometry has been apparent for quite some time. It has been recently proposed that a companion satellite equipped with a GNSS-R receiver could fly in formation with SMOS to provide sea surface roughness estimates (linked through the mss measurements) collocated both in time and space. In fact, the lack of reliable and colocated auxiliary fields for SMOS retrieval is the key motivation for this paper, which is a preliminary study of the potential application of GNSS-R signals in the framework of ocean salinity retrieval. Essentially, since SSS retrieval presents several pending issues, among the most significant is the adequate choice of the sea-state estimator, the purpose of this paper is to explore the capability of parameters extracted from GNSS-Rs to substitute for wind speed data in the sea-state correction.

This paper presents the results of a simulation study, and it is the natural continuation of a previous project performed by some of the authors for the ESA [21, WP1400]. 


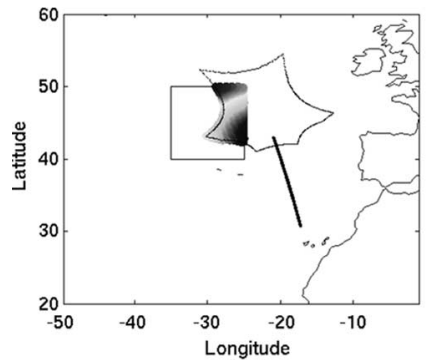

(a)

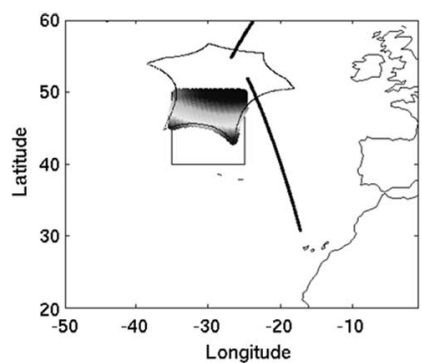

(b)
Fig. 1. Sample V-pol brightness temperatures for (a) ascending and (b) descending pass.

As a consequence, the methodology applied, the retrieval setup definition, and the assessment of results are oriented to the SMOS SSS retrieval framework.

The accuracy requirement for the retrieved SSS is 0.1 practical salinity units (psu) after monthly averaging over $2^{\circ} \times 2^{\circ}$ boxes.

This paper is divided into three main sections. Section II describes the simulation strategy, including the determination of the relationship between the mss auxiliary data and the wind speed, as well as the SSS retrieval setup. Section III presents and discusses the simulation results concerning the use of the global positioning system (GPS) constellation alone. Then, Section IV considers an extension of the study including other constellations suitable to be sources of GNSS-R signals. This paper ends with some conclusions and recommendations for further work in this field.

\section{Simulation Strategy}

The main steps in the simulation strategy are the following: generation of brightness temperatures in a selected test zone, computation of the specular points within the zone, identification of $\operatorname{mss}\left(U_{10}\right)$ relationship, association of the derived mss values, and SSS retrieval scheme definition.

\section{A. Brightness Temperature Generation}

In the framework of the ESA project [21], a representative mid-Atlantic test zone (longitude $35^{\circ} \mathrm{W}-25^{\circ} \mathrm{W}$, latitude $40^{\circ} \mathrm{N}-50^{\circ} \mathrm{N}$ ) was chosen for analysis since it exhibits rather homogeneous salinity but large wind speed variability.

Geocoded brightness temperatures were computed for both the ascending and descending passes by Institut Français de Recherche pour l'Exploitation de la Mer (IFREMER) for the whole month of January 2003, each time the simulated SMOS instantaneous FOV intersected the selected region of interest (ROI). These calculations were performed using the following auxiliary data [21, WP1100]: the blended QuikSCAT/NCEP wind product; the Centre de Météorologie Spatiale Satellite Application Facility/Ocean and Sea Ice (SAF/OSI) SST, and the World Ocean Atlas 2001 SSS climatological field, as geophysical inputs to the small scale approximation (SSA) for the sea surface L-band direct emission model [22]. Each time the SMOS' FOV crossed the selected zone, the auxiliary data closest in time were spatially resampled, and the corresponding brightness temperature fields at $\mathrm{H}$ and $\mathrm{V}$ polarizations were computed as described previously (Fig. 1). In our simulations, the brightness temperatures included the expected radiometric noise that will be present in SMOS measurements, including its variation within the SMOS FOV due to the antenna patterns and the so-called obliquity factor [23], [24]. These generated $T_{\mathrm{B}}$ 's were used as inputs for this paper.

\section{B. Estimation of the GNSS-R Derived mss}

There are two geometric invariants associated with the socalled mss tensor. One is $\sqrt{\sigma_{\mathrm{u}}^{2}+\sigma_{\mathrm{c}}^{2}}$, and the other one is 2 . $\sigma_{\mathrm{u}} \cdot \sigma_{\mathrm{c}}$, in which $\sigma_{\mathrm{u}}$ and $\sigma_{\mathrm{c}}$ are the sea surface slope standard deviation in upwind and crosswind directions, respectively.

The second invariant is chosen since it is the one defined in geometric optics theory, and it corresponds to the area of the "slope ellipse," although it degenerates in the case of a sinusoidal sea surface (ellipse completely flat). For the sea surface slope isotropy considered $\left(\sigma_{\mathrm{c}} / \sigma_{\mathrm{u}} \approx 0.7\right.$, according to the Elfouhaily spectrum [25]), the two definitions provide similar values.

In order to get the total mss $=2 \cdot \sigma_{\mathrm{u}} \cdot \sigma_{\mathrm{c}}$, the second moments of the Elfouhaily spectrum are computed. At L-band, the mss can be obtained by integrating the sea surface spectrum from a cutoff wavenumber defined as $2 \pi /(3 \lambda), \lambda$ being the electromagnetic wavelength. However, there is no unanimity concerning which cutoff wavenumber in the seasurface spectrum should be chosen in order to correctly reproduce the dependence of the L-band limited mss on wind speed, and different formulations can be found in the literature [12], [13], [26].

Moreover, it is expected that the mss actually measured will include other effects affecting the sea state, in addition to the wind speed.

Fig. 2(a) shows the optical Cox and Munk [27] mss in comparison to the total mss computed from Elfouhaily spectrum. Fig. 2(b) shows, in turn, the mss associated with the L1 GPS frequency $(\lambda=19 \mathrm{~cm})$. As is shown, not only is the value of the mss much smaller, but above $10-12 \mathrm{~m} / \mathrm{s}$, it decreases as about the logarithm of wind speed.

The GNSS-R simulated mss will then be obtained from the wind speed reference data (blended QuikSCAT/NCEP wind) with the fitting shown in Fig. 2(b), plus a given amount of noise assumed to be a zero-mean random Gaussian variable with a standard deviation equal to $0 \%, 5 \%$, or $10 \%$ of the mss value. Such errors are not the outcome of an mss error budget study, and no model for the statistics of mss retrievals has been considered [28], [29]. Instead, these thresholds were arbitrarily chosen, consistently with the purpose of this paper of finding out whether, assuming accurate mss fields, it is possible to fulfill and/or exceed the mission requirements.

\section{Identification of Specular-Reflection Points Within the ROI}

The specular-reflection points within the ROI defined by longitude $35^{\circ} \mathrm{W}-25^{\circ} \mathrm{W}$ and latitude $40^{\circ} \mathrm{N}-50^{\circ} \mathrm{N}$ (test zone), in which GNSS-Rs would have been collected by a hypothetical tandem SMOS satellite for the whole month of January 2003, have been calculated for each SMOS instantaneous FOV 


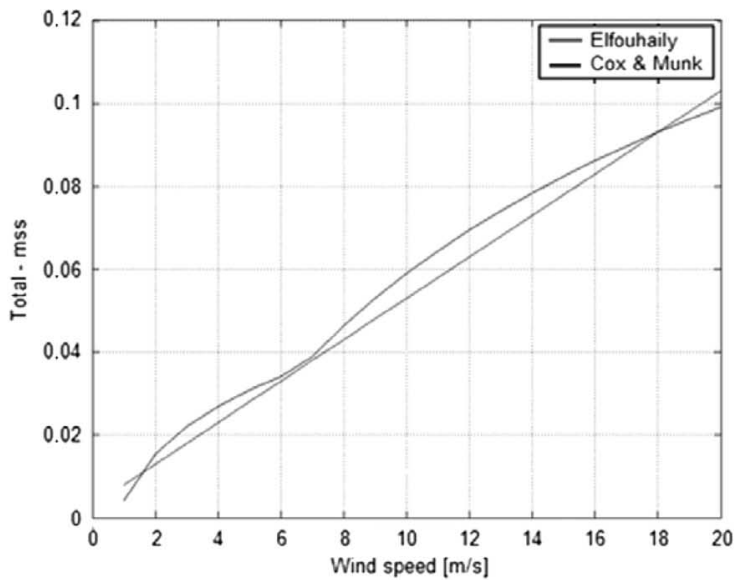

(a)

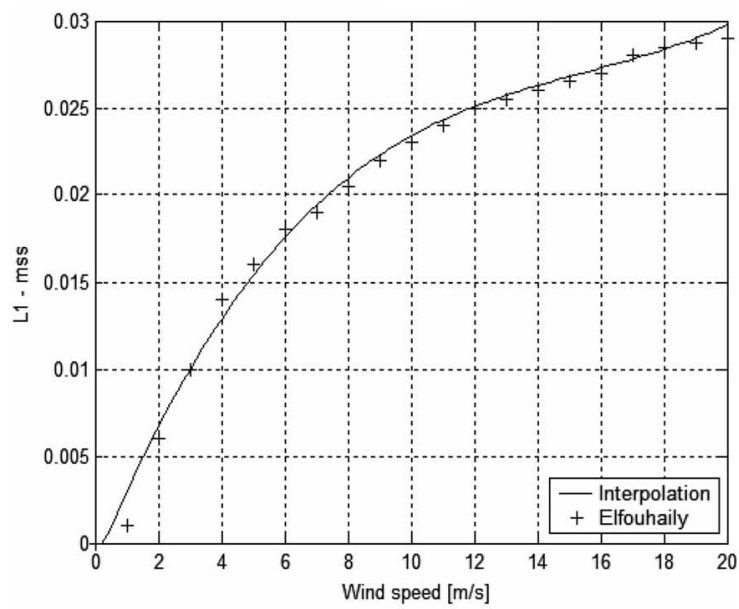

(b)

Fig. 2. (a) Cox and Munk [27] mss and total mss computed from the Elfouhaily spectrum without cutoff wavenumber. (b) MSS computed for the L1 GPS frequency $(\lambda=19 \mathrm{~cm})$.

(IFOV) of each day of the simulated month, providing hour, minute, and second and the latitude and longitude of each reflection. In a first configuration, the companion satellite was supposed to fly side-by-side in formation with SMOS, at same latitude and a different longitude. Fig. 3 presents two examples of specular points within the ROI for different passes on the same day.

Once geocoded to the icosahedron snyder equal area hexagonal grid of aperture 4 and resolution 9 (ISEA4H9) [30] to which SMOS data will be referred, these are the only points at which sea surface GNSS-R derived mss has been computed within the ROI and used as auxiliary data for the SSS retrieval algorithm.

\section{Estimation of the MSS at the Specular Points}

Using the blended QuikSCAT/NCEP wind speed reference data at the closest time to the tandem satellite passage and the $U_{10}$-mss relationship [Fig. 2(b)], the mss at the points of specular reflection within the ROI was estimated. Then, the generated brightness temperatures were associated with all the SMOS IFOVs in which these pixels are seen (Section II-A).

In this paper, the GNSS-R measurements are assumed to be made at the specular point. The inversion of GNSS-R measure-

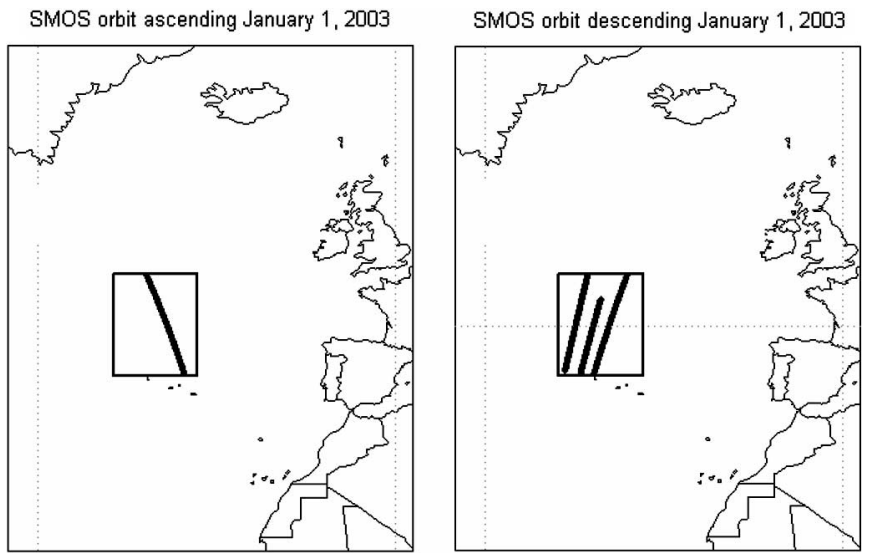

Fig. 3. Simulated specular points within the selected ROI for January 1, 2003. (left) Satellite ascending and (right) descending passes.

ments involves the fitting of a scattering model to the shape of a power-delay (correlation) waveform. The shape of this waveform represents the scattered power from a distributed area on the ocean surface, approximately in the shape of an ellipse with dimensions proportional to the square root of the delay.

However, in this study, the values of mss have been calculated from an analytical fitting with wind speed data and not by inversion of the waveform. As previously stated, to focus on the potential improvements in SMOS SSS retrievals from GNSS-R measurements, the processing of this signal has been left to be done separately, and mss values have been used as if they were the outcome of an established extraction procedure with a given error.

\section{E. SSS Retrieval in Terms of the mss at the Points of Specular Reflection}

The SSS retrieval scheme considered only the pixels for which a GNSS specular reflection exists.

As in the case when the SSS retrieval is formulated in terms of $U_{10}$ or the SWH, the SSS retrieval can now be formulated in the antenna $\left(T_{\mathrm{xx}}\right.$ and $\left.T_{\mathrm{yy}}\right)$ or in the Earth reference frames ( $T_{\mathrm{hh}}$ and $T_{\mathrm{vv}}$ ) [31], provided the appropriate corrections are applied (atmosphere, ionosphere, and sky downwelling radiation scattered over the Earth's surface). Both formulations require a precise knowledge of the observation geometry $(\Psi)$ and the Faraday rotation $\left(\Psi_{\text {Faraday }}\right)$ angles. Faraday rotation is not negligible at L-band and can exhibit significant inhomogeneities on the scale of $1 \mathrm{~km}$ within a SMOS pixel $(\geq 30 \mathrm{~km})$, which can jeopardize its correction. An alternative approach first proposed in [32] consists of formulating the retrieval problem in terms of the first Stokes parameter (I)

$$
I=T_{\mathrm{xx}}+T_{\mathrm{yy}}=T_{\mathrm{hh}}+T_{\mathrm{vv}}
$$

which is invariant to rotation (does not require a knowledge of the geometric and Faraday rotation angles), and the radiometric sensitivity is not degraded (antenna-Earth transformation matrix does not result in singularities). This formulation of the problem can be used in the dual-polarization mode in order to maximize the integration time, achieving better radiometric sensitivity than in the fully polarimetric mode. 
The process to retrieve the geophysical parameters is sketched in the following.

1) For each overpass, the error $\varepsilon$ (variance) between the model and the measured data at all incidence angles $\theta$ must be minimized to obtain a set of estimated parameters $(\hat{\vec{P}}=[\mathrm{SS} S, \mathrm{SS} \mathrm{T}, \mathrm{mŝs}])[31]$.

$$
\begin{aligned}
\varepsilon=\frac{1}{N_{\text {obs }}} \sum_{n}\{ & {\left[\bar{F}_{\text {model }}(\theta, \hat{\vec{P}})-\bar{F}_{\text {data }}(\theta, \vec{P})\right]^{T}(\overline{\bar{C}})^{-1} } \\
& \left.\times\left[\bar{F}_{\text {model }}(\theta, \hat{\vec{P}})-\bar{F}_{\text {data }}(\theta, \vec{P})\right]\right\}
\end{aligned}
$$

where $N_{\text {obs }}$ is the number of observations acquired from a single location in a satellite overpass, $\overline{\bar{C}}$ is the error covariance matrix that depends on the SMOS operation mode (fully polarimetric or dual polarization), the reference frame (Earth or antenna), and the pixel position in the FOV, and $\bar{F}_{\text {model } / \text { data }}(\theta, \hat{\vec{P}})$ is a vector that contains the modeled or the measured observables, and its structure depends on the formulation of the retrieval problem. Two different formulations are studied.

1) $\bar{F}(\theta, \vec{P})=\left[T_{\mathrm{hh}}(\theta, \vec{P}), T_{\mathrm{vv}}(\theta, \vec{P})\right]^{T}$, if the problem is formulated in terms of the brightness temperatures in the Earth's reference frame.

2) $\bar{F}(\theta, \vec{P})=[I(\theta, \vec{P})]^{T}=\left[T_{\mathrm{hh}}(\theta, \vec{P})+T_{\mathrm{vv}}(\theta, \vec{P})\right]^{T}=$ $\left[T_{\mathrm{xx}}(\theta, \vec{P})+T_{\mathrm{yy}}(\theta, \vec{P})\right]^{T}$, if the problem is formulated in terms of the first Stokes parameter.

A restrictive version of (2) includes cost terms for the variables to be retrieved

$$
\begin{aligned}
& \varepsilon=\frac{1}{N_{\text {obs }}} \sum_{n}\left\{\left[\bar{F}_{\text {model }}(\theta, \hat{\vec{P}})-\bar{F}_{\text {data }}(\theta, \vec{P})\right]^{T}(\overline{\bar{C}})^{-1}\right. \\
& \left.\times\left[\bar{F}_{\text {model }}(\theta, \hat{\vec{P}})-\bar{F}_{\text {data }}(\theta, \vec{P})\right]\right\} \\
& +\frac{\left(\mathrm{S} \hat{\mathrm{S} S}-\mathrm{SSS}_{\mathrm{ref}}\right)^{2}}{\sigma_{\mathrm{SSS}}^{2}}+\frac{\left(\mathrm{S} \hat{\mathrm{ST}}-\mathrm{SST}_{\mathrm{ref}}\right)^{2}}{\sigma_{\mathrm{SST}}^{2}} \\
& +\frac{\left(\mathrm{ms} s-\mathrm{mss}_{\mathrm{ref}}\right)^{2}}{\sigma_{\mathrm{mss}}^{2}} \text {. }
\end{aligned}
$$

$\mathrm{SSS}_{\mathrm{ref}}, \mathrm{SST}_{\mathrm{ref}}$, and $\mathrm{mss}_{\mathrm{ref}}$ are reference values (with their uncertainties) to be used to nudge the solution, and $\sigma_{\mathrm{SSS}}$, $\sigma_{\mathrm{SST}}$, and $\sigma_{\mathrm{mss}}$ are the corresponding auxiliary data standard deviations to properly weigh the cost function terms, according to the accuracy of the specific field.

However, it has been found that the reference value for SSS ( SSS $_{\text {ref }}$ ) tends to restrict the SSS solution too much to be near the value of $\mathrm{SSS}_{\text {ref }}$. Therefore, as concluded in [5] and [21], the constraint on SSS has not been included.

Finally, SSS retrievals on a pixel-by-pixel basis are temporally averaged for a whole month and spatially averaged in blocks of $1^{\circ} \times 1^{\circ}$ or $2^{\circ} \times 2^{\circ}$, in order to reduce the standard deviation of the estimates.

\section{Single GNSS-R Source Simulation Results}

With the objective of assessing the impact of different error sources, simulations have been performed at increasing complexity. In this section, only the retrieval using the first Stokes parameter computed in dual-pol mode is considered. Moreover, only the specular points calculated by means of the GPS constellation are used.

\section{A. SSS Retrieval in the Ideal Case}

An ideal scenario has been first built to test the robustness of the iterative method. This simulation was intended to test the reliability of the procedure despite the potential errors introduced by fitting the data either in the $\operatorname{mss}\left(U_{10}\right)$ conversion (due to the unavailability of "true" mss fields) or during the $U_{10}(\mathrm{mss})$ derivation (needed to establish a $T_{\mathrm{B}}(\mathrm{mss})$ geophysical model function in the inversion scheme). This method proved to be satisfactory, resulting in perfect SSS retrievals, apart from numerical round-off errors.

\section{B. SSS Retrieval With Radiometric Noise}

Second, a simulation that considered only the effect of the radiometric noise present in SMOS measurements was defined. The noise considered was $2.36 \mathrm{~K}$ at boresight, with a degradation factor toward the swath edges which is a function of the socalled "obliquity factor" and the antenna radiation patterns [23], [24]. The scope was to establish the best-case performance that an ideal instrument only limited by thermal noise could achieve in these conditions. The monthly rms error is nearly $0.47 \mathrm{psu}$, while the bias is negligible ( $0.013 \mathrm{psu})$.

\section{SSS Retrieval With GNSS-R Derived MSS Errors}

As previously discussed, salinity retrieval was studied assuming an uncertainty in the measured mss of 5\% and $10 \%$ of its value. In the beginning of this paper, $20 \%$ error was considered, but this resulted in unreasonable errors in retrieved SSS, due to the specific retrieval setup.

However, the use of a homogeneous mss value over the whole glistening zone might also be inaccurate, but to date, no GNSS-R system and algorithms have been proposed to overcome this issue.

Fig. 4(a) shows the inverse of Fig. 2(b) needed to use an mss field as input to the semiempirical model to correct for wind speed effects on $T_{\mathrm{B}}$. It is evident that a $10 \%$ error in the mss is equivalent to an error in $U_{10}$ of much larger than $10 \%$. This is made more explicit in Fig. 4(b), where for example, a 10\% error in mss is equivalent to a $1.5-\mathrm{m} / \mathrm{s} U_{10}$ error for $\mathrm{mss}=$ $0.020\left(U_{10}=7.6 \mathrm{~m} / \mathrm{s}\right)$, to a $0.7-\mathrm{m} / \mathrm{s} U_{10}$ error for $\mathrm{mss}=0.015$ $\left(U_{10}=4.6 \mathrm{~m} / \mathrm{s}\right)$, and to a $0.25-\mathrm{m} / \mathrm{s} U_{10}$ error at $\mathrm{mss}=0.010$ $\left(U_{10}=3 \mathrm{~m} / \mathrm{s}\right)$. Despite the fact that these numbers may seem reasonably accurate, the logarithmlike behavior in the mss in Fig. 2(b) strongly influences the variability of $U_{10}$ at high wind speeds. This is evident particularly above mss $=0.020-0.025$, i.e., above $U_{10} \sim 10 \mathrm{~m} / \mathrm{s}$, where a large percentage of wind speed data in the selected zone lies. Such effect will ultimately 


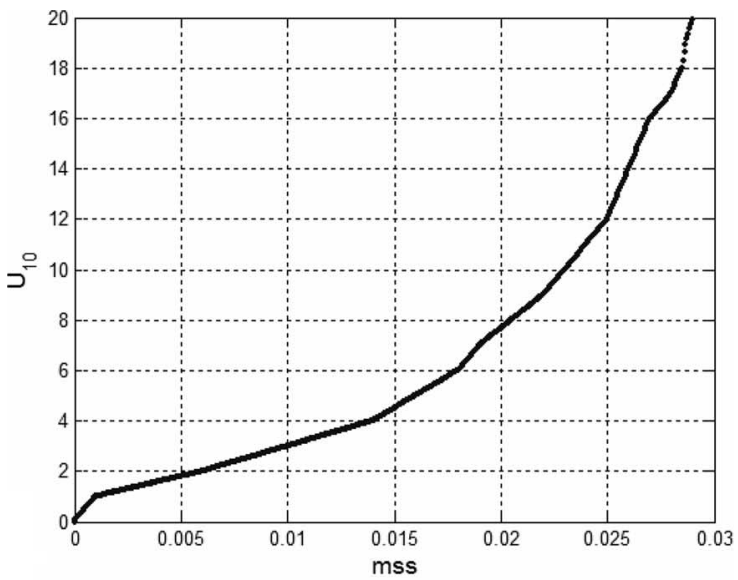

(a)

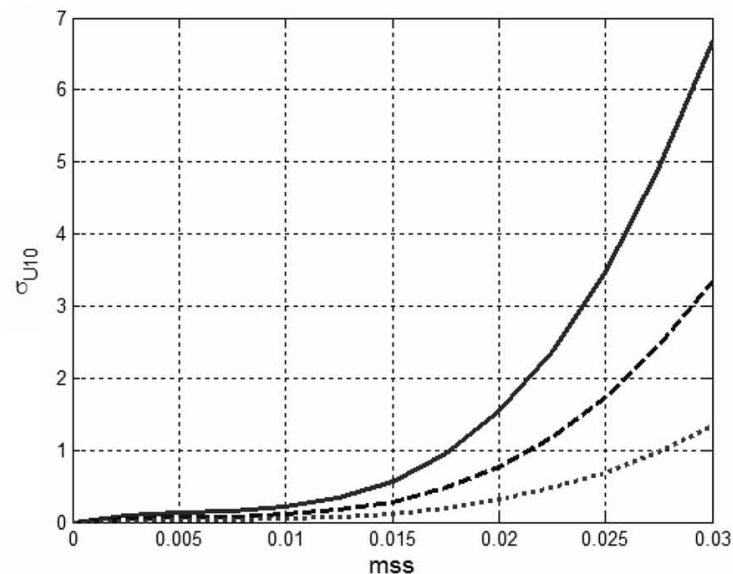

(b)

Fig. 4. (a) Wind speed versus mss. (b) $10 \%$ (solid line), $5 \%$ (dashed line), and $2 \%$ (dotted line) mss error propagation into $U_{10}$ error.

limit the retrieval capability, since the $T_{\mathrm{B}}(\mathrm{mss})$ relationship is derived from an existing $T_{\mathrm{B}}\left(U_{10}\right)$ model. In this paper, it has been assumed that the $T_{\mathrm{B}}(\mathrm{mss})$ dependence is given by $T_{\mathrm{B}}\left(U_{10}\right)$ from [33] and $U_{10}$ (mss) from Fig. 4(a).

This approach is suboptimal in two ways. First, it connects mss to $U_{10}$ in the case of GNSS-R, which is known to be an indirect link. Second, it connects $U_{10}$ to $T_{\mathrm{B}}$, which is another indirect link and not one-to-one, since $T_{\mathrm{B}}$ depends on other factors such as the SWH, wave age, and foam presence. Further efforts will have to be devoted, hence, to the definition of a better $T_{\mathrm{B}}$ (mss) model that would encompass the effects mentioned previously and that are implicitly considered within the "true" mss parameter.

In our case study, the input wind field exhibits the following statistics: $U_{10 \min }=0.02 \mathrm{~m} / \mathrm{s}, U_{10 \max }=33.7 \mathrm{~m} / \mathrm{s}, U_{10}$ avg $=$ $11.63 \mathrm{~m} / \mathrm{s}$, and $\sigma_{\mathrm{U} 10}=4.79 \mathrm{~m} / \mathrm{s}$. It is then clear that the sea state corresponding to the high winds encountered will be measured in most cases with a large uncertainty $\left(U_{10}\right.$ avg $=$ $11.63 \mathrm{~m} / \mathrm{s}$ corresponds to $\mathrm{mss}=0.0246$ ), which will increase salinity retrieval errors. That is, simulation results might be somewhat pessimistic, and better results could be obtained in other regions.

Fig. 5 shows the monthly errors (considering only the specular points) on a pixel-by-pixel basis and the corresponding

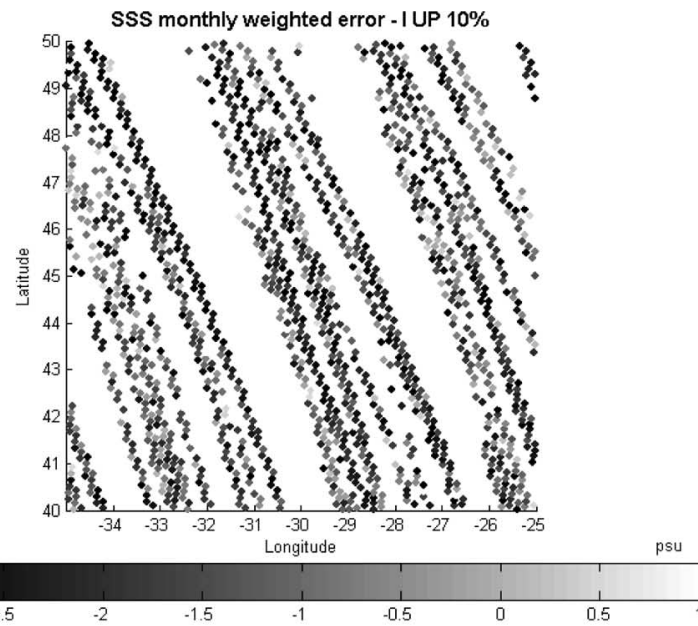

(a)

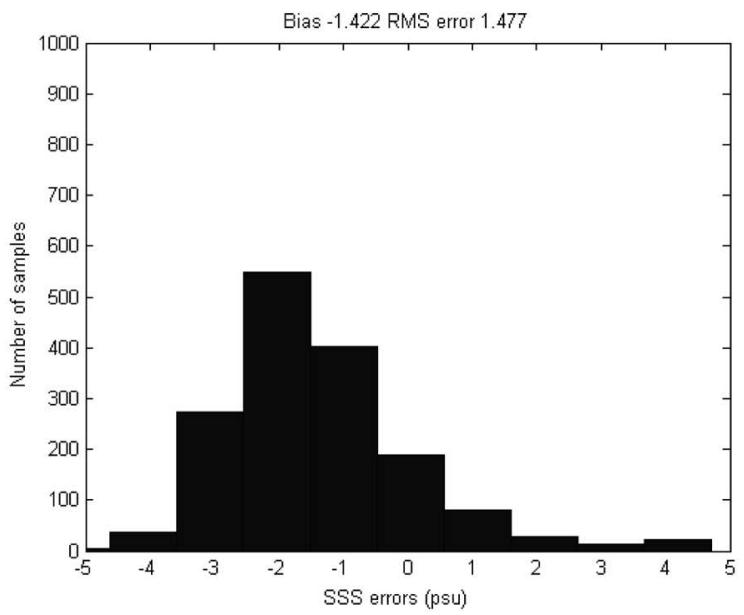

(b)

Fig. 5. (a) Monthly weighted errors in the ROI for specular points in dualpol mode for the ascending pass considering $10 \%$ mss error. (b) Histogram of corresponding monthly SSS weighted errors with bias and rms accuracy in the ROI.

histogram for those pixels for which a GPS specular reflection existed during the entire month. The retrieved SSS exhibits a -1.422-psu bias and a 1.477-psu standard deviation. The origin of this bias lies primarily in the difference between brightness temperature direct (SSA) and inverse [Hollinger's [33] plus $U_{10}$ (mss) dependence, Fig. 4(a)] models and also by the fact that a zero-mean Gaussian random error in mss does not correspond to a Gaussian random error in $U_{10}$. A similar effect was found in [5] when different $T_{\mathrm{B}}\left(U_{10}\right)$ models were used. Fig. 6 shows the monthly averaged errors in SSS considering a lower error $(5 \%)$ in mss field estimation. In this case, the retrieved SSS exhibits a -1.410 -psu bias, which is very similar to the previous case but a smaller standard deviation of $1.232 \mathrm{psu}$.

\section{Spatio-Temporal Averaging}

Temporal averaging was conducted with a proper weighted average procedure to give less weight to the noisier pixels that are farther away from the SMOS ground track. These weights were computed from the standard deviation of the retrievals 


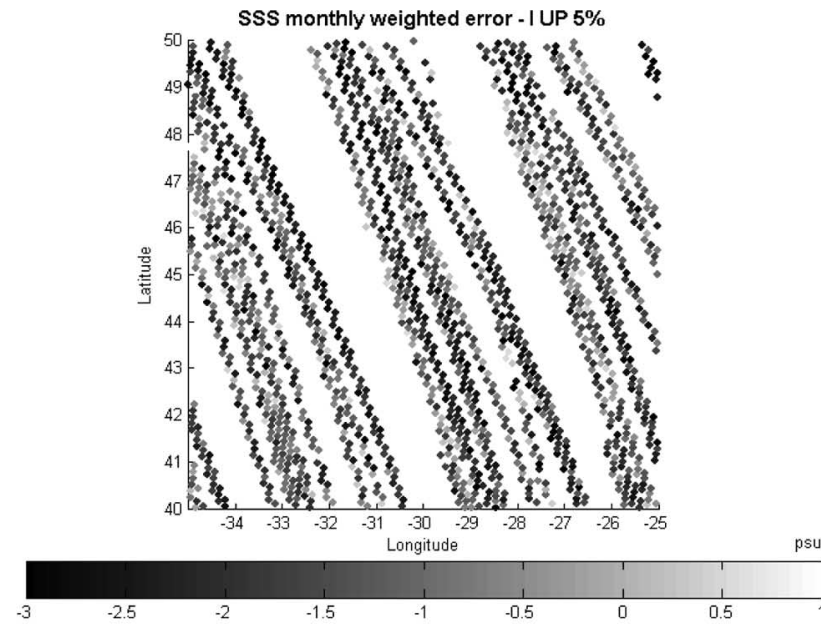

(a)

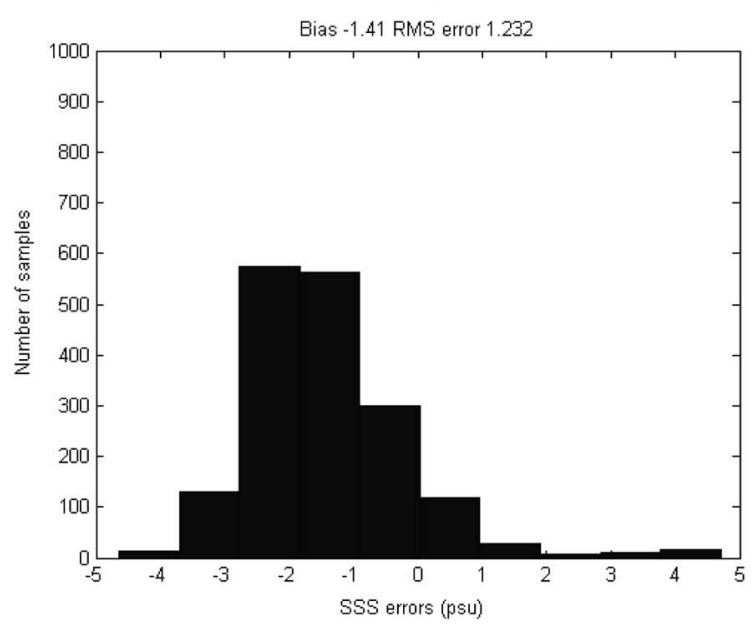

(b)

Fig. 6. Same as Fig. 5, for a 5\% mss error.

TABLE I

Single GNSS-R Source Simulation RESUlts

\begin{tabular}{|c|c|c|c|c|c|}
\hline Configuration & $\begin{array}{c}\text { Satellite } \\
\text { pass }\end{array}$ & $\begin{array}{c}\text { Monthy } \\
\text { Bias (psu) }\end{array}$ & $\begin{array}{l}\text { Monthly Rms } \\
\text { accuracy (psu) }\end{array}$ & $\begin{array}{c}R m s 1^{0} x 1^{o} \\
(p s u)\end{array}$ & $\begin{array}{c}\operatorname{Rms} 2^{o} \times 2^{o} \\
(p s u)\end{array}$ \\
\hline Radiometric Noise only & UP & 0.013 & 0.469 & 0.121 & 0.045 \\
\hline $5 \% m s s$ error & UP & -1.410 & 1.232 & 0.595 & 0.262 \\
\hline $10 \%$ mss error & UP & -1.422 & 1.477 & 0.724 & 0.315 \\
\hline
\end{tabular}

computed from ten Monte Carlo simulations. Spatial averaging was then performed over $1^{\circ} \times 1^{\circ}$ and $2^{\circ} \times 2^{\circ}$ boxes. The main results are shown in Table I. As is shown, due to the limited number of pixels with measured mss, the effectiveness of the spatial averaging is strongly jeopardized, limiting the expected improvement of the retrieval accuracies by using this kind of GNSS-R auxiliary data.

An obvious way to improve the SSS retrieval performance would be to increase the number of points in which the SSS can be retrieved within the ROI. To do this, an optimal interpolation and extrapolation procedure should be used, but none of the interpolation methods tested (nearest neighbor, bilinear, and MATLAB v4 interpolation techniques) have produced satisfactory results.

\section{Multiple GNSS-R Sources Simulation Results}

Once the limitations associated with the use of only GPS reflections and the spatial interpolation techniques have been shown, different (existing and future) GNSS constellations will be employed to improve spatio-temporal coverage using an optimal averaging strategy.

The constellations considered in this paper were the Russian GLONASS, the future European constellation GALILEO, in addition to points calculated from the satellite-based augmentation systems (SBAS)/INMARSAT telecommunications satellites in geostationary orbit.

In the previous configuration, the companion satellite would fly in formation side-by-side with SMOS. In addition, a second configuration has been studied, namely, considering a tandem satellite flying $500 \mathrm{~km}$ behind the SMOS payload in the same orbit.

In all cases in which mss errors greater than $5 \%$ were used, the resulting errors in the sea-state correction to $T_{\mathrm{B}}$ were found to be too large. Therefore, the following simulations were only conducted for the case of a 5\% error in mss. In other words, a best-case scenario concerning mss estimation was considered in a realistic and demanding SSS retrieval scheme.

The aim of this extension to the previous section is to verify the feasibility of blending information from different GNSS sources to achieve reasonable SSS accuracy. Thus, the overall setup of simulations consisted of four constellations, in two configurations with respect to SMOS (tandem satellite sideby-side and behind SMOS) for the two instrument operation modes ( $T_{\mathrm{hh}}$ and $T_{\mathrm{vv}}$ measured in fully polarimetric mode to avoid the singularities in the transformation from the antenna to the Earth's reference frame [31] or $I=T_{\mathrm{xx}}+T_{\mathrm{yy}}$ measured in dual-polarization mode) and for both satellite passes (ascending and descending). This setup resulted in 32 single-constellation simulations. Subsequently, to use as many specular-reflection points as possible, a grouping of the results from the previous simulations provided the final assessment of this paper. Table II summarizes the results of the single-constellation salinity retrieval in the different configurations mentioned previously.

The specular points from different constellations in both configurations exhibited a wide range of variability in their occurrence on the days considered. Namely, GPS and GALILEO had a larger number of days with available points in the side configuration than in the back one. Conversely, GLONASS and SBAS showed more specular points in the back configuration than in the side one. Concerning SBAS, specular-reflection points were available in both configurations only for the SMOS descending pass. In particular, in back configuration, there is a strong difference between the ascending passes (UP) and the descending ones (DN) regarding the number of points available on each day, particularly for the GPS constellation. A possible explanation for this effect is that when the companion satellite is behind SMOS in the ascending passes, it will capture specular reflections only from those GPS satellites present at those latitudes that are more "empty" than in the descending passes. This does not hold for GLONASS due to a more homogeneous distribution of the satellites generating reflections within the area under study. Hence, some of these results have to be 
TABLE II

Multiple GNSS-R SOURCES Simulation Results

\begin{tabular}{|c|c|c|c|c|c|c|c|c|}
\hline Constellation & Config. & Mode & Pass & $\begin{array}{c}\text { Monthly } \\
\text { Bias } \\
(p s u)\end{array}$ & $\begin{array}{c}\text { Monthly } \\
\text { Rms } \\
(p s u)\end{array}$ & $\begin{array}{c}1^{0} \times 1^{0} \\
(p s u)\end{array}$ & $\begin{array}{l}2^{\circ} \times 2^{\circ} \\
(p s u)\end{array}$ & $\begin{array}{c}\text { Number of } \\
\text { Days with } \\
\text { Points }\end{array}$ \\
\hline GPS & Side & I & UP & -1.410 & 1.232 & 0.595 & 0.262 & 25 \\
\hline GPS & Side & I & DN & -1.445 & 1.127 & 0.522 & 0.224 & 19 \\
\hline GPS & Side & ThTv & UP & -1.398 & 0.997 & 0.395 & 0.206 & 25 \\
\hline GPS & Side & ThTv & $\mathrm{DN}$ & -1.422 & 0.917 & 0.412 & 0.174 & 19 \\
\hline GLONASS & Side & I & UP & -1.646 & 1.295 & 0.611 & 0.313 & 23 \\
\hline GLONASS & Side & I & DN & -1.593 & 1.223 & 0.680 & 0.543 & 11 \\
\hline GLONASS & Side & ThTv & UP & -1.690 & 1.208 & 0.564 & 0.240 & 23 \\
\hline GLONASS & Side & ThTv & $\mathrm{DN}$ & -1.527 & 0.912 & 0.473 & 0.355 & 11 \\
\hline GALILEO & Side & I & UP & -1.660 & 1.158 & 0.492 & 0.276 & 24 \\
\hline GALILEO & Side & I & DN & -1.493 & 1.370 & 0.679 & 0.448 & 10 \\
\hline GALILEO & Side & ThTv & UP & -1.587 & 0.994 & 0.416 & 0.206 & 24 \\
\hline GALILEO & Side & ThTv & DN & -1.500 & 1.123 & 0.506 & 0.375 & 10 \\
\hline SBAS & Side & I & UP & - & - & - & - & 0 \\
\hline SBAS & Side & I & DN & -1.614 & 1.429 & 1.396 & 1.295 & 8 \\
\hline SBAS & Side & ThTv & UP & - & - & - & - & 0 \\
\hline SBAS & Side & ThTv & DN & -1.508 & 1.133 & 0.972 & 0.874 & 8 \\
\hline GPS & Back & I & UP & -1.913 & 1.016 & 0.693 & 0.560 & 3 \\
\hline GPS & Back & I & $\mathrm{DN}$ & -1.423 & 1.175 & 0.556 & 0.283 & 22 \\
\hline GPS & Back & ThTv & UP & -2.020 & 1.205 & 0.781 & 0.616 & 3 \\
\hline GPS & Back & ThTv & DN & -1.413 & 0.957 & 0.374 & 0.161 & 22 \\
\hline GLONASS & Back & I & UP & -1.907 & 1.130 & 0.829 & 0.635 & 14 \\
\hline GLONASS & Back & I & DN & -1.620 & 1.275 & 0.800 & 0.567 & 10 \\
\hline GLONASS & Back & ThTv & UP & -1.725 & 0.906 & 0.555 & 0.375 & 14 \\
\hline GLONASS & Back & ThTv & DN & -1.558 & 0.915 & 0.516 & 0.328 & 10 \\
\hline GALILEO & Back & I & UP & -1.654 & 1.229 & 0.771 & 0.346 & 4 \\
\hline GALILEO & Back & I & DN & -1.458 & 1.408 & 1.084 & 0.765 & 9 \\
\hline GALILEO & Back & ThTv & UP & -2.073 & 1.293 & 0.553 & 0.349 & 4 \\
\hline GALILEO & Back & ThTv & $\mathrm{DN}$ & -1.354 & 1.092 & 0.774 & 0.626 & 9 \\
\hline SBAS & Back & I & UP & - & - & - & - & 0 \\
\hline SBAS & Back & I & DN & -1.765 & 1.288 & 0.781 & 0.396 & 22 \\
\hline SBAS & Back & ThTv & UP & - & - & - & - & 0 \\
\hline SBAS & Back & ThTv & $\mathrm{DN}$ & -1.920 & 1.275 & 0.558 & 0.345 & 22 \\
\hline
\end{tabular}

Monthly Bias (psu)

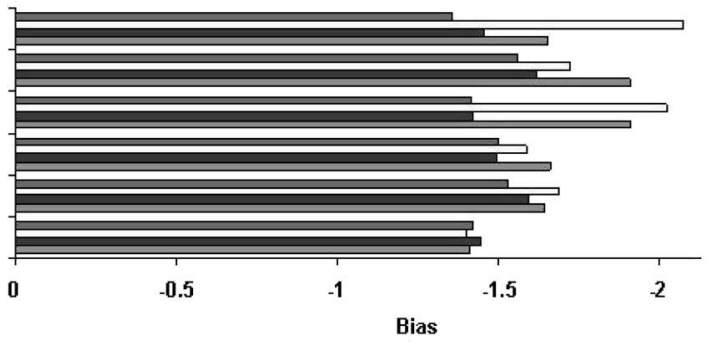

GALILEO back

GLONASS back

GPS back

GALILEO side

GLONASS side

GPS side

口IUP GIDN $\square$ THTV UP GThTVDN

Fig. 7. SSS monthly bias for different constellations (listed on the right side) and configurations (back and side) for both polarimetric modes (ThTV and I) and satellite passes (UP and DN).

interpreted carefully, since the number of days with specular points is limited, jeopardizing effective monthly averaging.

Figs. 7 and 8 show intercomparisons among the monthly biases in SSS error and the corresponding rms accuracy after $2^{\circ} \times 2^{\circ}$ spatio-temporal averaging, respectively.

Considering both ascending and descending passes, retrieved SSS rms accuracy turned out to be better for the GPS constellation in both side and back configurations. Conversely, GLONASS and GALILEO exhibited better retrievals for the ascending satellite passes. With respect to the accuracy of the

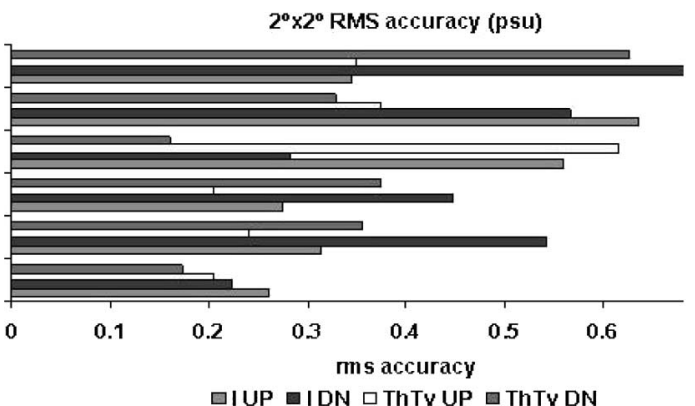

GALILEO back

GLONASS back GPS back

GALILEO side GLONASS side GPS side

Fig. 8. Spatio-temporally averaged $2^{\circ} \times 2^{\circ}$ SSS rms accuracy for different constellations and configurations (listed on the right side) and configurations (back and side) for both polarimetric modes (ThTv and I) and satellite passes (UP and DN).

two polarimetric modes, as in the case of a previous study [5], retrieval in fully polarimetric mode using $T_{\mathrm{hh}}$ and $T_{\mathrm{vv}}$ was better than using the first Stokes parameter in dual polarization.

The next step is to combine all the information in Table II to get a comprehensive retrieval, which considers all the specular points (with their corresponding mss fields) that could be collected in the SMOS FOV in the ROI on each specific day. As mentioned, the aim is to increase the number of specularreflection points available to perform better salinity retrieval after spatio-temporal averaging.

Thus, all the single-overpass SSS retrievals at the pixel level in the different configurations have been merged to obtain a retrieval for each day, and then, monthly averaging has been performed, as described in Section II. Pixels for which two or more SSS values from different constellations were simultaneously available have been averaged. Fig. 9(a)-(d) shows the monthly weighted errors in four cases for fully polarimetric mode, emphasizing the inhomogeneous sampling in the different configurations.

In most of the cases, even if the number of sampled pixels has definitely increased, the problem remains that many of them have never been imaged in the whole month or just a few times, hampering the averaging improvement. In spite of this, the blending of different GNSS-R derived mss data is however able to produce an improvement large enough to more nearly meet the global ocean data assimilation experiment (GODAE) requirements (0.1-psu accuracy after spatio-temporal averaging) [34] in some cases, with the key advantage of not depending on the availability of simultaneous and collocated wind speed data (or any other appropriate sea-state descriptor).

Table III summarizes the results gathered in the merged constellations approach, for both polarimetric modes, ascending and descending passes, and side and back tandem satellite positions. As is evident, not all the configurations presented an effective improvement with respect to the GPS taken as the default control constellation. However, it should be noticed that the SSS retrieved accuracy in $2^{\circ} \times 2^{\circ}$ boxes is consistent with the previously obtained results [21], particularly when referring to auxiliary wind data different from those that generated brightness temperatures. In fact, when dealing with a quasi-realistic situation in which models to generate and invert the data are different, using either wind speed data or mss fields different from the original inputs provide similar SSS rms error results. 


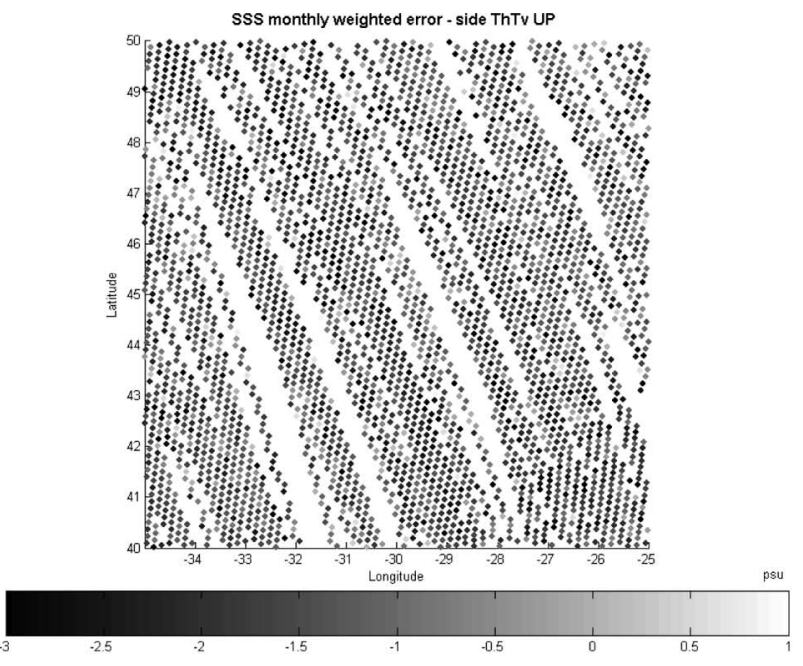

(a)

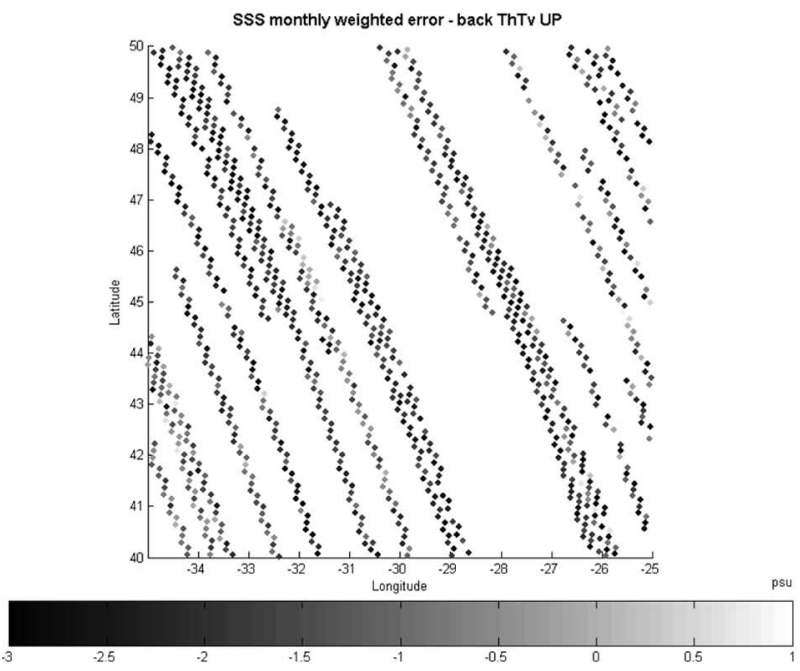

(c)

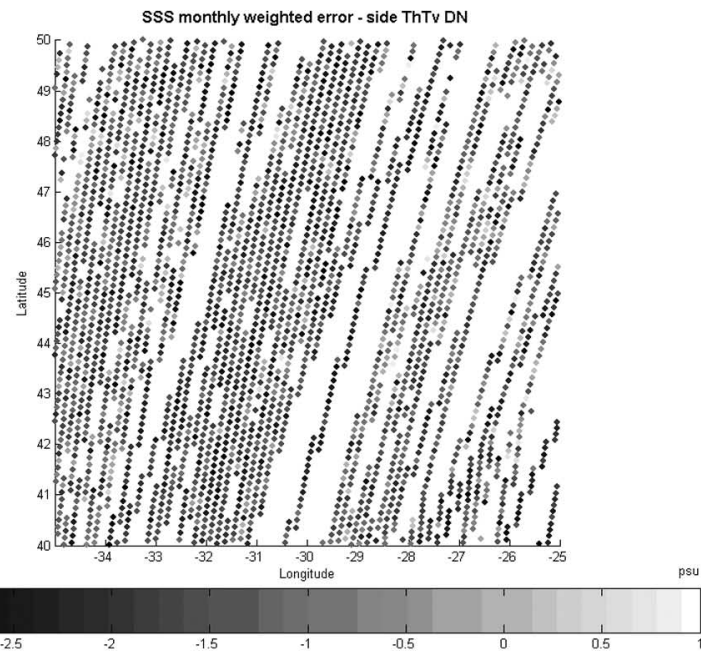

(b)

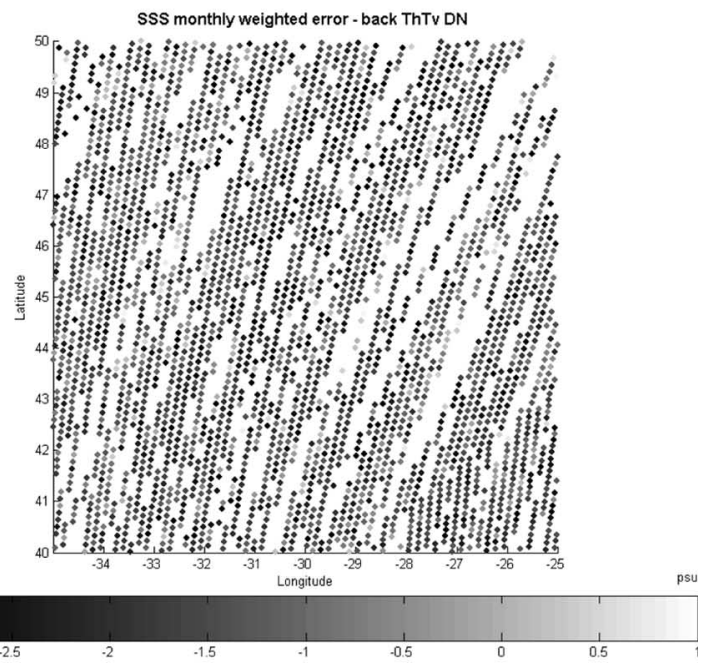

(d)

Fig. 9. Monthly weighted errors in the ROI for specular points for multiple blended constellations in fully polarimetric mode for (a) side tandem, ascending passes (b) side tandem, descending passes, (c) back tandem, ascending passes, and (d) back tandem, descending passes.

TABLE III

COUPLING OF GPS, GLONASS, GALILEO, AND SBAS CONSTELLATIONS

\begin{tabular}{|c|c|c|c|c|c|c|c|}
\hline Constellation & Configuration & Mode & Pass & $\begin{array}{c}\text { Monthly } \\
\text { Bias (psu) }\end{array}$ & $\begin{array}{c}\text { Monthly } \\
\text { Rms (psu) }\end{array}$ & $\begin{array}{l}1^{\circ} \times 1^{\circ} \\
(p s u)\end{array}$ & $\begin{array}{l}2^{0} \times 2^{\circ} \\
(p s u)\end{array}$ \\
\hline Multiple & Side & I & UP & -1.590 & 1.032 & 0.284 & 0.169 \\
\hline Multiple & Side & I & $\overline{\mathrm{DN}}$ & -1.507 & 1.131 & 0.453 & 0.308 \\
\hline Multiple & Side & ThTv & UP & -1.550 & 0.912 & 0.229 & 0.118 \\
\hline Multiple & Side & ThTv & $\mathrm{DN}$ & -1.463 & 0.879 & 0.303 & 0.210 \\
\hline Multiple & Back & I & UP & -1.882 & 1.122 & 0.722 & 0.558 \\
\hline Multiple & Back & I & $\mathrm{DN}$ & -1.540 & 1.153 & 0.467 & 0.292 \\
\hline Multiple & Back & ThTv & UP & -1.808 & 1.003 & 0.532 & 0.362 \\
\hline Multiple & Back & ThTv & $\mathrm{DN}$ & -1.547 & 0.999 & 0.307 & 0.176 \\
\hline
\end{tabular}

\section{CONCLUSION}

In this paper, the potential improvement by using GNSS-R opportunity signals as auxiliary data in the SSS retrieval procedure has been studied and evaluated. This analysis is the natural continuation of an ESA study aimed to stress the dependence of the retrieved SSS on the different auxiliary data (SST and $U_{10}$ ) used in the retrieval procedure.

Since neither the mss fields nor their relationship with other geophysical parameters describing the sea state (e.g., wind speed) currently exists, the mss values have been 
derived from the wind speed fields at the GNSS-R specularreflection points that a SMOS companion satellite flying sideby-side or $500 \mathrm{~km}$ behind SMOS would measure. These mss values have been used as auxiliary data in the SSS retrieval procedure.

The effectiveness of this auxiliary data set was limited by two main factors: the limited number of GNSS specular-reflection points in each overpass that does not allow efficient spatiotemporal averaging and the assumed large uncertainty in the sea state [in this case, parameterized in terms of $\operatorname{mss}\left(U_{10}\right)$ only] introduced by the nonlinearity of the mss at high $U_{10}$ values [Fig. 2(b)].

In order to overcome these problems, the mss fields cannot have an uncertainty larger than 5\% of the mss value, and several GNSS-R derived mss (from GPS, GLONASS, GALILEO, and SBAS) have been used to increase the number of specularreflection points. Simulation results show that the GODAE requirements can be nearly achieved in some specific configurations: side satellite, use of $T_{\mathrm{hh}}$ and $T_{\mathrm{vv}}$, and ascending passes.

Using GNSS-R signals seems then to provide unsatisfactory results, since the GODAE requirements are not accomplished except in one case, despite the small uncertainties assumed on mss retrieved with GNSS-R.

On the other hand, keeping this in mind, it has to be emphasized that one of the reasons of that is the coarse sampling of the zone, which is mostly due to the fact that only the specular point and not a larger glistening zone has been considered. In addition, the chosen SSS retrieval setup is itself constructed to achieve a quasi-realistic configuration, particularly considering that there were no restrictions on salinity.

Nevertheless, retrieved SSS values with multiple GNSS constellations are of the same order of results obtained in the aforementioned previous study [5], [21], whenever auxiliary winds are different from the original.

Future research might consider reflections not only in the exact moments that the specular reflections happen but within a narrow temporal window in which the sea state can be assumed to remain unchanged. The number of available mss points will thus be increased, and better results should be expected.

However, the advantage of GNSS-R signal lies in the spatiotemporal collocation of the measurements and on the expected improvement in the sampling on one hand (just described) and in the mss derivation on the other, thus justifying and motivating continued efforts in this direction.

Systematic measurements to determine the relationship between the $T_{\mathrm{B}}$ and the mss, along with other geophysical parameters such as $\mathrm{SWH}$, must be performed to improve the quality of the estimation of the mss and, thus, to be more valuable in the context of SMOS retrieval as well.

\section{ACKNOWLEDGMENT}

The authors would like to thank Dr. N. Reul (IFREMER) for providing the simulated brightness temperature data used in the study, Dr. O. Germain (Starlab), and J. F. Marchán (Polytechnic University of Catalonia) for providing valuable remarks during the revision of the paper and three anonymous reviewers for their comments and suggestions that helped improve the clarity of this paper.

\section{REFERENCES}

[1] SMOS: ESA's Water Mission, Jun. 14, 2004. BR-224. [Online]. Available: http://esamultimedia.esa.int/docs/br_224.pdf

[2] J. Font, G. Lagerloef, D. LeVine, A. Camps, and O. Z. Zanife, "The determination of surface salinity with the European SMOS space mission," IEEE Trans. Geosci. Remote Sens., vol. 42, no. 10, pp. 2196-2205, Oct. 2004.

[3] A. Camps, J. Font, M. Vall-llossera, C. Gabarró, I. Corbella, N. Duffo, F. Torres, S. Blanch, A. Aguasca, R. Villarino, L. Enrique, J. Miranda, J. Arenas, A. Juliá, J. Etcheto, V. Caselles, A. Weill, J. Boutin, S. Contardo, R. Niclós, R. Rivas, S. C. Reising, P. Wursteisen, M. Berger, and M. Martín-Neira, "The WISE 2000 and 2001 field experiments in support of the SMOS mission: Sea surface L-band brightness temperature observations and their application to multiangular salinity retrieval," IEEE Trans. Geosci. Remote Sens., vol. 42, no. 4, pp. 804-823, Apr. 2004.

[4] C. Gabarró, J. Font, A. Camps, M. Vall-llossera, and A. Juliá, "A new empirical model of sea surface microwave emissivity for salinity remote sensing," Geophys. Res. Lett., vol. 31, no. 1, p. L01309, Jan. 2004.

[5] R. Sabia, A. Camps, M. Vall-1lossera, and N. Reul, "Impact on sea surface salinity retrieval of different auxiliary data within the SMOS mission," IEEE Trans. Geosci. Remote Sens., vol. 44, no. 10, pp. 2769-2778, Oct. 2006.

[6] M. Martin-Neira, "A passive reflectometry and interferometry system (PARIS): Application to ocean altimetry," ESA J., vol. 17, no. 4, pp. 331$355,1993$.

[7] M. Martin-Neira, P. Colmenarejo, G. Ruffini, and C. Serra, "Ocean altimetry using the carrier phase of GNSS reflected signals," in Proc. Ocean Winds, Plouzane, France, Nov. 2000. CERSAT News, Issue 11.

[8] M. Belmonte Rivas and M. Martin-Neira, "Coherent GPS reflections from the sea surface," IEEE Geosci. Remote Sens. Lett., vol. 3, no. 1, pp. 28-31, Jan. 2006

[9] F. Soulat, M. Caparrini, O. Germain, P. Lopez-Dekker, M. Taani, and G. Ruffini, "Sea state monitoring using coastal GNSS-R," Geophys. Res. Lett., vol. 31, no. 21, L21303, 2004.

[10] G. Ruffini, M. Caparrini, B. Chapron, F. Soulat, O. Germain, and L. Ruffini, "Oceanpal: An instrument for remote sensing of the ocean and other water surfaces using GNSS reflections," in Proc. EuroGOOS, Athens, Greece, Dec. 3-6, 2002, pp. 146-153.

[11] J. L. Garrison and J. L. Katzberg, "Effects of sea roughness on bistatically scattered range coded signals from the global positioning system," Geophys. Res. Lett., vol. 25, no. 13, pp. 2257-2260, 1998.

[12] A. Komjathy, V. Zavorotny, P. Axelrad, G. H. Born, and J. L. Garrison, "GPS signal scattering from sea surface: Wind speed retrieval using experimental data and theoretical model," Remote Sens. Environ, vol. 73, no. 13, pp. 162-174, 2000.

[13] J. L. Garrison, A. Komjathy, V. U. Zavorotny, and S. J. Katzberg, "Wind speed measurement using forward scattered GPS signals," IEEE Trans. Geosci. Remote Sens., vol. 40, no. 1, pp. 50-65, Jan. 2002.

[14] E. Cardellach, G. Ruffini, D. Pino, A. Rius, A. Komjathy, and J. L. Garrison, "2003 Mediterranean Balloon Experiment: Ocean wind speed sensing from the stratosphere, using GPS reflections," Remote Sens. Environ., vol. 88, no. 3, pp. 351-362, Dec. 2003.

[15] S. Lowe, C. Zuffada, Y. Chao, P. Kroger, J. L LaBreque, and L. E. Young, "5-cm precision aircraft ocean altimetry using GPS reflections," Geophys. Res. Lett., vol. 29, no. 10, pp. 4359-4362, May 2002.

[16] A. Rius, J. M. Aparicio, E. Cardellach, M. Martín-Neira, and B. Chapron, "Sea surface state measured using GPS reflected signals," Geophys. Res. Lett., vol. 29, no. 23, p. 2122, 2002.

[17] G. Ruffini, F. Soulat, M. Caparrini, O. Germain, and M. MartinNeira, "The Eddy Experiment: Accurate GNSS-R ocean altimetry from low altitude aircraft," Geophys. Res. Lett., vol. 31, no. 12, L12306, Jun. 2004.

[18] O. Germain, G. Ruffini, F. Soulat, M. Caparrini, B. Chapron, and P. Silvestrin, "The Eddy Experiment: GNSS-R speculometry for directional sea-roughness retrieval from low altitude aircraft," Geophys. Res. Lett., vol. 31, no. 21, L21307, 2004. DOI: 10.1029/2004GL020991.

[19] S. Lowe, J. LaBrecque, C. Zuffada, L. J. Romans, L. E. Young, and G. A. Hajj, "First spaceborne observation of an Earth-reflected GPS signal," Radio Sci., vol. 37, no. 1, p. 7-1, Feb. 7, 2002.

[20] S. Gleason, S. Hodgart, S. Yiping, C. Gommenginger, S. Mackin, M. Adjrad, and M. Unwin, "Detection and processing of bistatically reflected GPS signals from low Earth orbit for the purpose of ocean remote sensing," IEEE Trans. Geosci. Remote Sens., vol. 43, no. 6, pp. 12291241, Jun. 2005. 
[21] Synergetic aspects and auxiliary data concepts for sea surface salinity measurements from space, Jan. 2006. [Online]. Available: ftp://ftp. estec.esa.nl/pub/vr/SMO/Salinity_Synergy_18176_FR.pdf

[22] N. Reul and B. Chapron, "SMOS-Salinity data processing study improvements in emissivity models," IFREMER, France, WP 1100 Report, Oct. 2001. ESA Contract No. 15165/01/NL/SF.

[23] A. Camps, I. Corbella, J. Bará, and F. Torres, "Radiometric sensitivity computation in aperture synthesis interferometric radiometry," IEEE Trans. Geosci. Remote Sens., vol. 35, no. 2, pp. 680-685, Mar. 1998.

[24] A. Camps, I. Corbella, J. Bará, and F. Torres, "Corrections to radiometric sensitivity computation in aperture synthesis interferometric radiometry," IEEE Trans. Geosci. Remote Sens., vol. 36, no. 5, p. 1835, Sep. 1998.

[25] T. Elfouhaily, B. Chapron, K. Katsaros, and D. Vandermark, "A unified directional spectrum for long and short wind-driven waves," J. Geophys. Res., vol. 102, no. C7, pp. 15781-15796, 1997.

[26] D. R. Thompson, T. M. Elfouhaily, and J. L. Garrison, "An improved geometrical optics model for bistatic GPS scattering from the ocean surface," IEEE Trans Geosci. Remote Sens., vol. 43, no. 12, pp. 2810-2821, Dec. 2005.

[27] C. Cox and W. Munk, "Measurements of the roughness of the sea surface from photographs of the sun's glitter," J. Opt. Soc. Amer., vol. 44, no. 11, pp. 838-850, Nov. 1954.

[28] C. Zuffada and V. U. Zavorotny, "Coherence properties of the GPS signal scattered off the ocean the accuracy of remote sensing of sea surface," in Proc. IGARSS, 2001, pp. 3332-3334.

[29] H. You, J. L. Garrison, G. Heckler, and V. U. Zavorotny, "Stochastic voltage model and experimental measurement of ocean-scattered GPS signal statistics," IEEE Trans Geosci. Remote Sens., vol. 42, no. 10, pp. 21602169, Oct. 2004.

[30] Discrete Global Grids (DGGs)_Icosahedral Snyder Equal Area (ISEA) projection. [Online]. Available: http://www.sou.edu/cs/sahr/dgg/ index.html

[31] A. Camps, M. Vall-llossera, N. Duffo, F. Torres, and I. Corbella, "Performance of sea surface salinity and soil moisture retrieval algorithms with different auxiliary datasets in 2-D L-band aperture synthesis interferometric radiometers," IEEE Trans. Geosci. Remote Sens., vol. 43, no. 5, pp. 1189-1200, May 2005.

[32] A. Camps, I. Corbella, M. Vall-llossera, N. Duffo, F. Torres, R. Villarino, L. Enrique, F. Julbé, J. Font, A. Juliá, C. Gabarró, J. Etchetto, J. Boutin, A. Weill, V. Caselles, E. Rubio, P. Wursteisen, and M. Martín-Neira, "L-band sea surface emissivity: Preliminary results of the WISE-2000 campaign and its application to salinity retrieval in the SMOS mission," Radio Sci., vol. 38, no. 4, 8071, Jun. 19, 2003. DOI: 10.1029/ 2002RS002629.

[33] J. P. Hollinger, "Passive microwave measurements of sea surface roughness," IEEE Trans. Geosci. Electron., vol. GE-9, no. 3, pp. 165-169, Jul. 1971.

[34] N. Smith and M. Lefébvre, "The Global Ocean Data Assimilation Experiment (GODAE)," in Proc. Int. Symp. Monitoring Oceans 2000s: An Integr. Approach, Biarritz, France, Oct. 15-17, 1997.

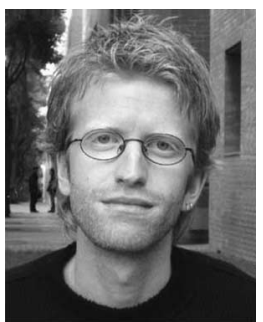

Roberto Sabia (S'03) was born in Naples, Italy, on November 13, 1975. He received the degree in marine environmental sciences (cum laude), a curriculum in oceanography (five-year course study), from the Università degli Studi di Napoli "Parthenope," Naples, in 2002. He is currently working toward the $\mathrm{Ph} . \mathrm{D}$. degree in signal theory and communications at the Polytechnic University of Catalonia (UPC), Barcelona, Spain.

In 2002, he was a recipient of a grant on sea surface scattering models at the Microwave Remote Sensing Laboratory, Università degli Studi di Napoli "Parthenope." In 2003, he was with the Department of Signal Theory and Communications, UPC. He participated in the FROG 2003, REFLEX 2003, and MOUSE 2004 field campaigns and participated in two European Space Agency studies within the frame of the Soil Moisture and Ocean Salinity Earth Explorer Mission. In 2006, he spent four months in the Ocean Observing and Climate Department, National Oceanography Centre, Southampton, U.K., working on salinity retrieval at different spatial resolution scales. His main research interests deal with microwave radiometry and sea surface salinity retrieval.

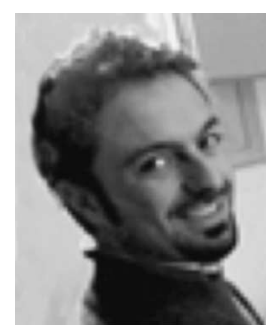

Marco Caparrini received the Laurea degree in electronic engineering (spec. remote sensing) in 1995 from the University of Rome "La Sapienza," Rome, Italy.

His main research field is the use of global navigation satellite system (GNSS) signals as a source of opportunity for remote sensing of planet Earth (PARIS concept). In particularly, he concentrates his efforts in the development of novel algorithms for code and phase altimetry with GNSS-reflection signals, backing the development of new instruments able to exploit this new concept. Currently, he is with Starlab, Barcelona, Spain.

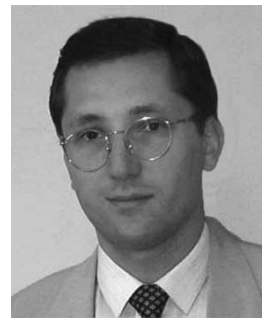

Adriano Camps (S'91-A'97-M'00-SM'03) was born in Barcelona, Spain, in 1969. He received the degree in telecommunications engineering and the $\mathrm{Ph} . \mathrm{D}$. degree in telecommunications engineering from the Polytechnic University of Catalonia (UPC), Barcelona, Spain, in 1992 and 1996, respectively. From 1991 to 1992, he was with the ENS des Télécommunications de Bretagne, Bretagne, France, with an Erasmus Fellowship. In 1993, he was with the Electromagnetics and Photonics Engineering Group, Department of Signal Theory and Communications, UPC, as an Assistant Professor, and since 1997, he has been an Associate Professor. In 1999, he was on sabbatical leave at the Microwave Remote Sensing Laboratory, University of Massachusetts, Amherst. His research interests are microwave remote sensing with special emphasis on microwave radiometry by aperture synthesis techniques. He has performed numerous studies within the frame of the ESA's SMOS Earth Explorer Mission. He is an Associate Editor of Radio Science.

Dr. Camps received the second national award of university studies in 1993, the INDRA award from the Spanish Association of Telecommunication Engineering to the best Ph.D. in 1997, the extraordinary Ph.D. award from the Universitat Politècnica de Catalunya in 1999, the First Duran Farell Award, and the Ciudad de Barcelona Award, in 2000 and 2001, respectively, both for technology transfer, and the Research Distinction of the Generalitat de Catalunya for contributions to microwave passive remote sensing in 2002 . $\mathrm{He}$ also won the "Premi Nacional de Telecomunicacions 2003," "Premi Salvà i Campillo 2004," and the "European Young Investigator" Award in 2004. He was the Chair of $\mu \mathrm{Cal}$ ' 01 . He has been the Editor of the IEEE GEOSCIENCE AND Remote SENSING Newsletter and President-Founder of the IEEE Geoscience and Remote Sensing Society Chapter in Spain.

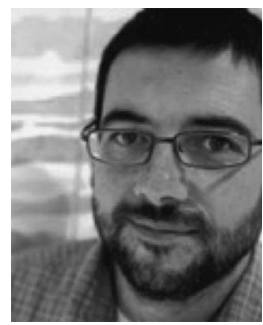

Giulio Ruffini graduated from the University of California, Berkeley, in mathematics and physics and received the Ph.D. degree in physics from the University of California, Davis.

$\mathrm{He}$ is currently CEO of Starlab, Barcelona, Spain, a company that identifies business and science opportunities in order to create disruptive technologies for the emerging space and applied neuroscience markets. 